evaluated. Furthermore, pre-treatment structures in the microbiome were compared with structures 1 week and 2 months after treatment.

Results Alpha diversity analysis showed that both richness and evenness were recovered to pre-treatment levels at 2 months after eradication therapy. There were almost no differences in bacterial species abundance between pre- and post-treatment samples in beta diversity analysis. Although the relative abundance of Bacteroidetes tended to increase and Actinobacteria significantly decreased immediately after eradication, the taxonomic composition was similar to that pre-eradication at 2 months post-eradication. However, 2 students showed significant structural changes in their relative abundances at the phylum level.

Conclusions This study suggests a limited impact of $H$. pylori eradication therapy on the gut microbiome in healthy midteenagers. However, we should continuously monitor the effects of changes to the gut microbiome caused by antibiotic use.

\section{IDDF2018-ABS-0055 THE NATURAL HISTORY OF TERMINAL ILEAL RESECTION IN CROHN'S DISEASE}

Jonathan Segal ${ }^{*}$, Alan Askari, Susan Clark, Ailsa Hart, Omar Faiz. St Mark's Hospital, UK

10.1136/gutjnl-2018-IDDFbestabstracts. 12

Background CD can affect any part of the GI tract, but the terminal ileum (TI) is the most common. The risk of surgery based on all population-based studies, at 1,5 , and 10 years after diagnosis of $\mathrm{CD}$ is $16.3 \%, 33.3 \%$ and $46.6 \%$ respectively. The risk of reoperation following a TI resection has been reported as $70 \%$ at 20 years based on a population of 1379 patients. A recent study comparing laparoscopic ileal resection versus infliximab found that laparoscopic ileocaecal resection results in similar quality-of-life scores and is not associated with more serious adverse. The long-term outcomes of those undergoing ileal resection have yet to be studied and may help identify risk factors for those that may require further operations.

Methods The Hospital Episodes Statistics (HES) dataset was used to identify all patients admitted to hospitals in England from 1997-2012. Time trends and multivariate logistic regression analysis was undertaken to determine factors associated with repeated resection.

Results 155236 patients were identified as having CD with a median follow-up was 92 months (IQR 43-143). Of these, $18.8 \%(29,257)$ underwent TI resection. Amongst this subgroup, $11.0 \% \quad(n=3,101 / 29,257)$ required more than one resection. The total number of procedures performed was 32,889 , of which $56.6 \%(\mathrm{n}=18,622 / 32,889)$ were right hemicolectomy/ileo-caecal resection. The median time between resections was 41 months (IQR 15-74 months). There was no significant change in the number of procedures performed each year $(\mathrm{p}=0.693)$. Of the total population, $7.8 \%$ $(n=12,173 / 155,236)$ had Infliximab therapy.

Conclusions Nearly one in five patients will require surgical intervention over a 15 year period for $\mathrm{CD}$, of those undergoing TI resection only $11 \%$ of these will require further surgery. This is the largest population-based study reporting the requirement for further surgery following TI resection and suggests that the reoperation rate is far lower than previously reported. The number of procedures performed has largely remained the same over time suggesting that medical therapy has not altered the overall rates of further surgery following TI surgery in CD.

\section{IDDF2018-ABS-0073 DIFFERENCES WITH EXPERIENCED NURSE ASSISTANCE DURING COLONOSCOPY IN DETECTING POLYP AND ADENOMA: A RANDOMISED CLINICAL TRIAL}

${ }^{1}$ Weihong Wang*, ${ }^{2} \mathrm{Lu} \mathrm{Xu}$, 'Zhenfei Bao, 'Linyin Sun, ${ }^{1}$ Chunyan Hu, ${ }^{1}$ Feng Zhou, ' ${ }^{1}$ Lei Xu. ${ }^{1}$ Department of Gastroenterology, Ningbo First Hospital, China; ${ }^{2}$ Ningbo University Medical School, China

\subsection{6/gutjnl-2018-IDDFbestabstracts. 13}

Background This study aims to evaluate whether the participation of an experienced endoscopy nurse in colonoscopy increases the polyp detection rate (PDR) and adenoma detection rate (ADR) of experienced colonoscopists.

Methods This study was a randomised controlled trial (Clinicaltrials.gov No. NCT02292563). Patients were randomly assigned to the experienced colonoscopist alone (single observer) group or experienced nurse participation (dual observer) group. The primary outcome was the PDR and ADR. The advanced lesions detection rate was also recorded.

Results A total of 587 patients were included in the analysis. Among these patients, 291 patients were assigned to the single observer group, while 296 patients were assigned to the dual observer group. The PDR was $33 \%$ in the single observer group and $41.9 \%$ in the dual observer group $(p=0.026)$, while the ADR was $23.0 \%$ in the single observer group and $30.4 \%$ in the dual observer group $(p=0.043)$. No significant difference was found for advanced lesions between groups.

Conclusions The present data demonstrated that experienced nurse observation during colonoscopy could improve polyp and adenoma detection rates, even if the colonoscopist is experienced.

\section{IDDF2018-ABS-0076 OPTIMISED 14-DAY LEVOFLOXACIN SEQUENTIAL VERSUS 10-DAY BISMUTH QUADRUPLE THERAPY CONTAINING HIGH DOSE ESOMEPRAZOLE IN THE SECOND- LINE AND THIRD-LINE TREATMENT OF HELICOBACTER PYLORI - A MULTICENTER RANDOMISED TRIAL}

${ }^{1}$ Jyh-Ming Liou*, ${ }^{1}$ Chieh-Chang Chen, ${ }^{2}$ Po-Yueh Chen, ${ }^{3} Y u$-Jen Fang, ${ }^{4}$ Jaw-Town Lin, ${ }^{1}$ Ming-Shiang Wu. 'Department of Internal Medicine, National Taiwan University Hospital, Taiwan; ' 2 Department of Internal Medicine, Chia-Yi Christian Hospital, Taiwan; ${ }^{3} 3$ Department of Internal Medicine, National Taiwan University Hospital, Yun-Lin Branch, Taiwan; ${ }^{4}$ School of Medicine, Fu Jen Catholic University, Taiwan

\subsection{6/gutjnl-2018-IDDFbestabstracts. 14}

Background We aimed to compare the efficacy of 14 day levofloxacin sequential therapy versus 10 day bismuth quadruple therapy in the second-line and third-line treatment of Helicobacter pylori (H. pylori) infection.

Methods $H$. pylori infected patients who failed after one treatment were eligible in this open labelled, multicenter, randomised trial, and were randomised to receive (1) levofloxacin sequential therapy (EAML): esomeprazole $40 \mathrm{mg}$ and amoxicillin $1 \mathrm{~g}$ for the first 7 days, followed by esomeprazole $40 \mathrm{mg}$, metronidazole $500 \mathrm{mg}$, and levofloxacin $250 \mathrm{mg}$ for another 7 days (all twice daily); or (2) bismuth quadruple therapy (BQ): esomeprazole 